\title{
Situational awareness: a tacit yet viable concept
}

\author{
Avi Parush, PhD
}

Received: 14 May 2017/Accepted: 19 May 2017/Published online: 2 June 2017

(C) Canadian Anesthesiologists' Society 2017

Most of us are familiar with the term situational awareness (SA), and some may even have an intuitive grasp of iti.e., "to know what is going on". Nevertheless, once we dig deeper into another's familiarity with the concept, the full understanding is often quite limited. Furthermore, SA rarely translates well into daily practice.

Such is the key message of the report by Haber et al. in this issue of the Journal. In addressing anesthesiologists' understanding of SA, ${ }^{1}$ they suggest that anesthesiologists are familiar with the concept of SA, yet it is generally silently understood. This tacit characteristic is due in part to the lack of an operational definition which could be made more explicit during clinical training.

In our own needs assessment, ${ }^{2}$ which Haber $e$ t al. cite to support their own article, we also found that key informants considered SA a highly important concept, yet most indicated that it is not sufficiently taught to trainees. What then are the challenges associated with SA? In this editorial, I extend the discussion of these challenges by a) defining SA as a more explicit, complex, context-sensitive concept and $b$ ) applying this broadened understanding to approaches for teaching SA as a learnable skill set.

Accordingly, my main thesis is twofold. First, the lack of an operational definition of SA is linked to it being a theoretically challenged concept. Specifically, its definition is somewhat nebulous with ill-defined conceptual borders,

A. Parush, $\mathrm{PhD}(\square)$

Israel Institute of Technology, Technion City, Haifa, Israel

e-mail: aparush@technion.ac.il; aviparush@gmail.com

A. Parush, $\mathrm{PhD}$

Department of Psychology, Carleton University, Ottawa, ON, Canada and it is also highly contextual. Second, SA is nevertheless a viable concept that we as practitioners can apply.

\section{Some theoretical challenges of the SA concept}

Construct and process

The literature refers to SA as an ill-defined concept, ${ }^{3}$ challenged by being variably defined as a construct, process, ability, or skill, or most likely some combination of these terms. It is further inadequately theorized because it also incorporates several key perceptual, attentional, and cognitive processes. This makes SA less focused and harder to test. Mica Endsley made a serious initial attempt to define SA and put forward a theoretical model ${ }^{4}$ by describing its various process levels. Level 1 encompasses perceptual and attentional processes (in addition to working memory) required for the acquisition of information and gathering facts. Levels 2 and 3 encompass cognitive constructs such as long-term memory and processes that include understanding and thinking ahead. These considerations suggest that SA is either a sophisticated knowledge structure or a collection of processes with which we perform situation assessment to reach an "awareness" outcome.

Not well bounded

There is often confusion between SA Level 3 (as defined by Endsley) and decision-making and choice of action. ${ }^{5}$ In SA Level 3, we project into the future and assess what may happen given the facts and our interpretation. But sometimes the assessment of what may happen depends on what we might do in the given situation. For example, 
we could engage in a "what if" kind of assessment in order to think ahead. Such thinking mentally simulates possible actions that could influence the situation. Moreover, it casts doubt on whether this reasoning is SA Level 3 or insight beyond SA altogether.

\section{Highly contextual}

Another interesting challenge associated with the SA concept is that the relevant elements vary depending on the situation. The concept originally evolved within the aviation domain where spatial and temporal elements are critical. When we look into a healthcare-related situation, we see that it shares some situational aspects with aviation, especially the temporal ones. Conversely, spatial awareness is less critical and actually rare in the context of healthcare. In other words, SA is a highly contextual concept, which makes it difficult to determine "good SA" - i.e., the scale of good or poor SA depends on the context. Consequently, it is difficult to develop "standardized" tools and methods to measure SA. The specific metrics we can use to measure SA also depend on the context.

\section{The viability of the SA concept}

Regardless of these theoretical challenges, the SA concept has been considered and studied empirically for almost three decades. One way to reconcile these theoretical challenges is to think of SA as an umbrella or metaconstruct. ${ }^{6}$ In other words, SA can be viewed as an aggregate of perceptual and cognitive processes aimed at assessing a given situation. Despite the risk that an umbrella concept might be hard to study and measure, SA has shown high construct validity. ${ }^{5}$ For example, we could find a correlation between SA scores and experience levels, ${ }^{7}$ implying that the concept is robust enough to reflect expected differences. Viewing it as an umbrella concept with high construct validity makes the SA concept very useful and viable.

Herein lies a significant opportunity to translate SA into practice through training. Situational awareness as an umbrella concept consists of various perceptual and cognitive processes which are trainable skills. ${ }^{8}$ Thus, a possible approach to teaching $\mathrm{SA}$ is to emphasize the components of the process within the context of relevant tasks and situations. For example, training for a particular task, such as a handover, could focus on the use of working or running memory within the overall need to maintain SA. Also, training for anesthesiologists' tasks during surgery could focus on attentional control within the overall need to maintain SA. Specifically, anesthesiologists are required to allocate their attentional resources to multiple tasks and information sources-i.e., their own tasks and other tasks and events that take place in the operating room. Such attentional control is a trainable skill.

\section{One last remark}

Haber et al. include a quote in their article that starts with "Driving, for example...", reflecting how anesthesiologists may understand SA using examples from everyday life (e.g., driving a car). While SA is highly contextual and should be trained as such, we can and should learn from other situations and domains, such as driving and aviation where there is much to learn. This type of application can help make the SA concept more explicit and operational in healthcare contexts. While SA is tacit as a concept due to its shaky theoretical foundation, it is nevertheless a useful concept and can be trained as a specific skill set.

\section{La conscience situationnelle : un concept à la fois tacite et viable}

Bien que la conscience situationnelle soit un terme que la plupart d'entre nous connaissons (et certains en possédant même une définition intuitive, c.-à-d. "savoir ce qui se passe »), si l'on cherche à creuser sous la familiarité apparente de tout un chacun avec ce concept, on se rend rapidement compte que sa compréhension est bien souvent limitée. Qui plus est, la conscience situationnelle est difficile à bien traduire dans la pratique quotidienne.

Voilà le message clé du compte rendu de Haber et coll. dans ce numéro du Journal, qui traite de la compréhension de la conscience situationnelle par les anesthésiologistes. ${ }^{1}$ Selon cette étude, les anesthésiologistes connaissent bien le concept, quoiqu'il soit en général compris de façon silencieuse. Cet aspect tacite du concept est d'une part lié à l'absence de définition fonctionnelle, et d'autre part au fait qu'il pourrait être plus explicité pendant la formation.

Dans notre propre étude d'évaluation des besoins, ${ }^{2}$ que Haber et coll. citent pour appuyer leurs travaux, nous avons nous aussi observé que les informateurs clés estimaient que la conscience situationnelle était un concept très important; toutefois, la plupart ont indiqué qu'il n'était pas suffisamment enseigné aux stagiaires. Pourquoi ce concept représente-t-il donc un tel défi? Dans cet éditorial, j'aimerais prolonger la discussion au sujet des défis de la conscience situationnelle afin de : (a) définir la conscience situationnelle en tant que concept plus explicite, plus complexe et plus sensible au contexte; et (b) appliquer cette compréhension élargie aux approches 
d'enseignement de la conscience situationnelle en tant que compétence qui peut être acquise.

Ainsi, mon propos principal comporte deux volets. En premier lieu, l'absence de définition fonctionnelle de la conscience situationnelle est liée au fait qu'il s'agit d'un concept controversé d'un point de vue théorique - plus spécifiquement, sa définition est quelque peu nébuleuse et ses limites conceptuelles mal définies; de plus, le concept est très fortement lié au contexte. Deuxièmement, la conscience situationnelle est tout de même un concept viable avec lequel nous pouvons, en tant que groupe de praticiens, travailler.

\section{Le concept de conscience situationnelle et ses défis théoriques}

\section{Le construit et le processus}

La littérature fait référence à la conscience situationnelle en tant que concept mal défini ${ }^{3}$ et problématique. En effet, il est parfois décrit comme un construit, parfois comme un processus, une capacité ou une compétence ou, ce qui est le plus fréquent, comme une combinaison de tous ces éléments. La conscience situationnelle est également mal théorisée parce qu'elle intègre plusieurs processus perceptuels, attentionnels et cognitifs clés. Ainsi, le concept est moins clair et plus difficile à mettre à l'épreuve. Dans la première tentative sérieuse de définir la conscience situationnelle et de proposer modèle théorique, 4 Mica Endsley décrit les divers niveaux de processus du concept. Le premier niveau recoupe les processus perceptuels et attentionnels, ainsi que la mémoire à court terme, nécessaire à l'acquisition de l'information et la collecte de faits. Les niveaux 2 et 3 englobent les construits cognitifs tels que la mémoire à long terme et les processus qui recoupent la compréhension et l'anticipation (ou la projection dans le futur). Ces considérations laissent à penser que la conscience situationnelle est soit une structure de connaissance sophistiquée, soit une collection de processus avec lesquels nous évaluons la situation, résultant en une «conscience » de ladite situation.

\section{Un concept mal délimité}

Il y a souvent confusion entre le niveau 3 de conscience situationnelle (telle que définie par Endsley) et la prise de décision et les choix d'action. ${ }^{5}$ Dans le niveau 3 de conscience situationnelle, l'individu se projette dans le futur et évalue ce qui pourrait arriver en se fondant sur les faits et leur interprétation. Toutefois, il arrive que l'évaluation de ce qui pourrait arriver dépende de ce que l'individu pourrait faire dans une situation donnée. Par exemple, on peut s'adonner à une évaluation de type « et si...? » afin de réfléchir à l'avenir et de l'anticiper. Une telle façon de penser simule mentalement les actions possibles qui pourraient influencer la situation. Ainsi, s'agit-il vraiment du niveau 3 de conscience situationnelle, ou de quelque chose qui se situe complètement au-delà de la conscience situationnelle?

Un concept extrêmement contextuel

Un autre défi intéressant lié au concept de conscience situationnelle est que les éléments pertinents varient selon la situation. À l'origine, le concept a vu le jour et évolué dans le monde de l'aviation. Dans ce domaine, les paradigmes spatiotemporels à même une situation donnée sont cruciaux. En ce qui a trait au domaine des soins de santé, certains aspects situationnels présents dans le monde de l'aviation s'y retrouvent également, particulièrement les aspects temporels. En revanche, la conscience spatiale est moins cruciale et, en fait, plutôt rare dans un contexte de soins de santé. La conscience situationnelle est donc un concept très fortement lié au contexte. Cela rend d'autant plus ardue la définition d'une «bonne conscience situationnelle », car une bonne ou une mauvaise conscience situationnelle sera toujours fonction du contexte. Par conséquent, il est difficile de mettre au point des outils et des méthodes standardisés pour mesurer la conscience situationnelle. Les mesures spécifiques pour évaluer la conscience situationnelle sont également dépendantes du contexte.

\section{Viabilité du concept de conscience situationnelle}

Indépendamment des défis théoriques qui l'entourent, le concept de conscience situationnelle fait depuis près de trente ans l'objet de réflexions et d'études empiriques. Une façon de réconcilier ces défis théoriques et la conscience situationnelle serait d'y penser comme un terme générique, ou un méta-construit. ${ }^{6}$ En d'autres mots, la conscience situationnelle peut être envisagée comme un agrégat de processus perceptuels et cognitifs dont le but est d'évaluer une situation donnée. Malgré le risque qu'un concept générique soit difficile à étudier et à mesurer, la conscience situationnelle a démontré une validité de construit élevée. ${ }^{5}$ Par exemple, nous pourrions trouver une corrélation entre les scores de conscience situationnelle et les niveaux d'expérience, $^{7}$ ce qui suggère que le concept est suffisamment robuste pour refléter les différences attendues. En l'imaginant comme un concept générique disposant d'une grande validité de construit, le concept de conscience situationnelle devient alors très utile et viable. 
Et c'est là que repose l'occasion d'appliquer la conscience situationnelle à la pratique par la formation. La conscience situationnelle, en tant que concept générique, se compose de divers processus perceptuels et cognitifs, lesquels sont des compétences qui peuvent être acquises. ${ }^{8}$ Dès lors, une approche possible pour enseigner la conscience situationnelle serait d'attirer l'attention sur ses composantes de processus dans le contexte de tâches et de situations pertinentes. Par exemple, nous pourrions enseigner des tâches tels que les transferts en faisant ressortir l'utilisation de la mémoire à court terme ou de la mémoire de travail tout gardant à l'esprit la nécessité de maintenir la conscience situationnelle. Un autre exemple serait d'enseigner les tâches des anesthésiologistes pendant la chirurgie en mettant l'accent sur le contrôle de l'attention tout en maintenant la conscience situationnelle. Plus spécifiquement, les anesthésiologistes doivent allouer leurs ressources d'attention à de nombreuses tâches et sources d'informations : leurs propres tâches, mais aussi les autres tâches et événements qui ont lieu dans la salle d'opération. Un tel contrôle de l'attention est une compétence qui peut être acquise.

\section{Dernières considérations}

L'une des citations dans l'article de Haber et coll. commence ainsi : «Conduire, par exemple...». Cet exemple reflète bien la façon dont certains anesthésiologistes appréhendent la conscience situationnelle, soit à l'aide d'exemples de la vie quotidienne (par ex., le fait de conduire son auto). Alors que la conscience situationnelle est fortement liée au contexte et devrait être enseignée de cette manière, nous pouvons - et devrions - nous inspirer d'autres situations et d'autres domaines. La conduite et l'aviation sont des domaines qui ont beaucoup à nous apprendre; cela pourrait rendre le concept de conscience situationnelle plus explicite et fonctionnel dans le contexte des soins de santé. Bien que le concept de conscience situationnelle soit tacite en raison de ses fondements théoriques nébuleux, il s'agit toutefois d'un concept utile qui peut être enseigné sous forme de compétence spécifique.

Conflicts of interest None declared.

Editorial responsibility This submission was handled by Dr. Hilary P. Grocott, Editor-in-Chief, Canadian Journal of Anesthesia.

\section{Conflit d'intérêt Aucun.}

Responsabilité éditoriale Cet article a été traité par Dr Hilary P. Grocott, rédacteur en chef, Journal canadien d'anesthésie.

\section{References}

1. Haber J, Ellaway R, Chun R, Lockyer J. Exploring anesthesiologists' understanding of situational awareness: a qualitative study. Can J Anesth 2017; 64. doi:10.1007/s12630-017-0904-2

2. Parush A, Campbell C, Hunter A, et al. Situational Awareness and Patient Safety. Ottawa, ON, Canada. The Royal College of Physicians and Surgeons of Canada; 2011. Available from URL: https://www. editorialmanager.com/caan/download.aspx $? \mathrm{id}=100247 \&$ guid $=\% 7 \mathrm{~B} 8$ 214955B-901A-4D08-A653-8DB6F396F064\%7D\&scheme=1 (accessed May 2017).

3. Sarter NB, Woods DD. Situation awareness: a critical but illdefined phenomenon. Int J Aviation Psychol 1991; 1: 45-57.

4. Endsley MR. Toward a theory of situation awareness in dynamic systems. Human Factors 1995; 37: 32-64.

5. Wickens $C D$. Situation awareness: review of Mica Endsley's 1995 articles on situation awareness theory and measurement. Human Factors 2008; 50: 397-403.

6. Sarter NB, Woods $D D$. How in the world did I ever get into that mode: mode error and awareness in supervisory control. Human Factors 1995; 37: 5-19.

7. Crozier MS, Ting HY, Boone DC, et al. Use of human patient simulation and validation of the Team Situation Awareness Global Assessment Technique (TSAGAT): a multidisciplinary team assessment tool in trauma education. J Surg Educ 2015; 72: 156-63.

8. Gopher D. Emphasis change as a training protocol for high demands tasks. In: Kramer AF, Weigman DA, Kirlik A (Eds). Attention: From Theory to Practice. Oxford Psychology Press; 2007: 209-24. 